\title{
Inhalt.
}

IV. Benzolderivate und heterocyklische Verbindungen.

Benzolderivate.

Seite

Allgemeines . . . . . . . . . . . . . . . . . . . . . 3

A. Kohlenwasserstoffe . . . . . . . . . . . . . . . . . . . . . 8

326. Cymol („Camphen", „Camphogen" [1)UMas], „Cymen" [Gerhardi und CABodrs], -p-Methyl-isopropyl-benzol . . . . . . . . . . . . . 8

m-Cymol . . . . . . . . . . . . . . . . . . . . . . . 23

327. Styrol . . . . . . . . . . . . . . . . . . . . . . . . . 24

328. Naphtalin . . . . . . . . . . . . . . . . . . . . . . . 28

329. Sequojen . . . . . . . . . . . . . . . . . . . . . . . 29

B. Substitutionsprodukte der Kohlenwasserstoffe . . . . . . . . . . 31

I. Nur im Kern substituierte Kohlenwasserstoffe, also Phenole und ihre

Äther bzw. Ester. . . . . . . . . . . . . . . . . . . . 31

a) Einwertige Phenole und ibre Derivate . . . . . . . . . . 34

$\alpha_{1}$ ) Einwertige Phenole von Benzolkoblenwasserstoffen mit gesättigten Seitenketten und ihre Derivate . . . . . . . . 34

330. p-Kresol . . . . . . . . . . . . . . . . . . . . . . . . 34

331. m-Kresol . . . . . . . . . . . . . . . . . . . . . . . . 35

332. Methyläther des p-Kresols . . . . . . . . . . . . . . . . . . . . 36

333. Acetyl-p-Kresol . . . . . . . . . . . . . . . . . . . . . 36

334. Methyläther eines Phlorols . . . . . . . . . . . . . . . . . 37

335. Carvacrol . . . . . . . . . . . . . . . . . . . . . . . . 38

336. Thymol . . . . . . . . . . . . . . . . . . . . . . . . 51

$\alpha_{2}$ ) Einwertige Phenole von Benzolkohlenwasserstoffen mit ungesättigter Seitenkette und ihre Derivate . . . . . . . . 64

337. Chavikol . . . . . . . . . . . . . . . . . . . . . . . . 66

338. Methylchavikol, Isoanethol, Estragol . . . . . . . . . . . . . . 69

339. Anethol . . . . . . . . . . . . . . . . . . . . . . . . 76

Anetholnitrit . . . . . . . . . . . . . . . . . . . . . . 88

Anetholnitrosochlorid . . . . . . . . . . . . . . . . . . 90

ß) Zweiwertige Phenole und ihre Derivate (Äther) . . . . . . . 92

$\beta_{1}$ ) Zweiwertige Phenole von Benzolkohlenwasserstoffen mit gesättigten Seitenketten und ihre Derivate . . . . . . . . 92

o-Diphenole. . . . . . . . . . . . . . . . . . . . . . 92

340. Guajakol . . . . . . . . . . . . . . . . . . . . . . . . 92

m-Diphenole. . . . . . . . . . . . . . . . . . . . . . 92

p-Diphenole. . . . . . . . . . . . . . . . . . . . . . . 92

341. p-Hydrochinonäthyläther . . . . . . . . . . . . . . . . . . . 93

342. Thymobydrochinon . . . . . . . . . . . . . . . . . . . . . . . 93

343. Dimethyläther des Thymohydrochinons . . . . . . . . . . . . . . 94 
$\beta_{2}$ ) Zweiwertige Phenole von Benzolkohlenwasserstoffen mit ungesättigten Seitenketten und ihre Derivate . . . . . . . 94

344. Betelphenol, Chavibetol . . . . . . . . . . . . . . . . . . 96

345. Eugenol . . . . . . . . . . . . . . . . . . . . . . . . 100

Alkyl- und Acylderivate des Eugenols, die sich als Bestandteile ätherischer Öle finden . . . . . . . . . . . . . . 117

346. Methyleugenol $=$ Methylbetelphenol . . . . . . . . . . . . . . 117

Säurederivate des Eugenols . . . . . . . . . . . . . . 123

347. Aceteugenol . . . . . . . . . . . . . . . . . . . . . . 123

348. Acetylsalicylsäureester des Eugenols . . . . . . . . . . . . . 125

349. Isoeugenol . . . . . . . . . . . . . . . . . . . . . . . 126

350. Methylisoeugenol . . . . . . . . . . . . . . . . . . . . 137

351. Safrol . . . . . . . . . . . . . . . . . . . . . . . . 139

Isosafrol = (1)-Propenyl-(3,4)-oxymethylenbenzol . . . . . . . . . . 147

r) Dreiwertige Phenole und ihre Derivate . . . . . . . . . 155

$\gamma_{1}$ ) Dreiwertige Phenole von Benzolkohlenwasserstoffen, die keine ungesättigte Seitenkette haben, und ihre Derivate 155

352. Dimethyläther des Pyrogallols. . . . . . . . . . . . . . . . 155

$\gamma_{2}$ ) Dreiwertige Phenole von Benzolkohlenwasserstoffen mit ungesätigter Seitenkette und ihre Derivate. . . . . . . . 156

353. Asaron (Asarin, Asarit, Haselwurzkampfer) . . . . . . . . . . . 157

354. Myristicin . . . . . . . . . . . . . . . . . . . . . . . 165

Isomyristicin . . . . . . . . . . . . . . . . . . . . . . 169

§) Vierwertige Phenole und ihre Derivate . . . . . . . . . . 172

355. Petersilienapiol, Petersilienkampfer $\mathrm{C}_{12} \mathrm{H}_{14} \mathrm{O}_{4}$ (Petroselinol) . . . . . 172

Isapiol . . . . . . . . . . . . . . . . . . . . . . . . 180 Verhalten des Apiols und Isapiols gegen $\mathrm{N}_{2} \mathrm{O}_{3}$. . . . . . . . . . . . 183

356. Dillapiol . . . . . . . . . . . . . . . . . . . . . . . 185

Dillisapiol = (1)-Propenyl-(3,4)-oxymethylen-(5,6)-dioxymethylbenzol . . 187

8) Sonstige Phenole bzw. phenolartige Körper, die sich als Bestandteile ätherischer öle finden . . . . . . . . . . . 192

II. Substitutionsprodukte der Kohlenwasserstoffe, sofern sie Benzolderivate sind, mit Substituenten in der Seitenkette, jedoch ohne glelchzeitige Substitution im Kern dureh OH bzw. Oxalkyl oder Oxyalkylen . 195

a) Alkohole. . . . . . . . . . . . . . . . . . . . . . . 195

a $)$ Gesättigte Alkohole. . . . . . . . . . . . . . . . . . 195

357. Benzylalkohol . . . . . . . . . . . . . . . . . . . . . . 196

358. Phenyläthylalkohol . . . . . . . . . . . . . . . . . . . . 204

Phenylpropenylalkohol . . . . . . . . . . . . . . . . . . 207

a) Ungesättigte Alkobole . . . . . . . . . . . . . . . . 208

Zimtalkohol . . . . . . . . . . . . . . . . . . . . . 208

b) Aldehyde . . . . . . . . . . . . . . . . . . . . . . 208

$b_{1}$ ) Gesättigte Aldehyde. . . . . . . . . . . . . . . . . 208

359. Benzaldehyd . . . . . . . . . . . . . . . . . . . . . 208

360. Cuminaldehyd . . . . . . . . . . . . . . . . . . . 217

361. Hydrozimtaldehyd . . . . . . . . . . . . . . . . . . . . 224

bq) Ungesättigte Aldehyde . . . . . . . . . . . . . . . . 225

362. Zimtaldehyd . . . . . . . . . . . . . . . . . . . . 225 


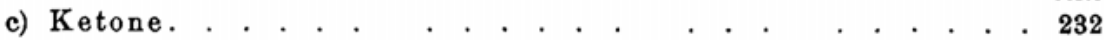

Acetophenon . . . . . . . . . . . . . . . . . . . . . . . . . . . . .

d) Säuren . . . . . . . . . . . . . . . . . . . . . . . . . . . . . . . . . . . . .

di) Gesättigte Săuren . . . . . . . . . . . . 233

363. Benzoësäure . . . . . . . . . . . . . . . . . . . . . . . . . 233

$\mathrm{d}_{2}$ ) Ungesättigte Säuren . . . . . . . . . . . . . . . . . . . . . . . 237

364. Zimtsäure . . . . . . . . . . . . . . . . . . . . . . . 237

III. Substitutionsprodukte der Kohlenwasserstofe, soweit sie Benzolderivate sind, mit Substituenten in der Seitenkette und gleichzeitiger Substitution im Kern dureh OH, Oxalkyl oder Oxalkylen usw. 241

a) Alkohole. . . . . . . . . . . . . . . . . . . . . . . 241

b) Aldehyde . . . . . . . . . . . . . . . . . . . . . . 241

$b_{1}$ ) Monooxyaldehyde und ihre Ätherderivate . . . . . . . . 242

Einwertige Aldehyde. . . . . . . . . . . . . . . . . 242

365. o-Oxybenzaldehyd, Salicylaldehyd; salicylige, spiroylige Säure . . . . 242

p-Oxybenzaldehyd . . . . . . . . . . . . . . . . . . . . 249

366. Alkylierter einwertiger Aldehyd. p-Methoxybenzaldehyd, Anisaldehyd . 251

$\mathrm{b}_{2}$ ) Zweiwertige Aldehyde. . . . . . . . . . . . . . . . 256

367. Vanillin . . . . . . . . . . . . . . . . . . . . . . . . 256

368. Piperonal (Heliotropin) . . . . . . . . . . . . . . . . . . 265

$b_{8}$ ) Dreiwertige Aldehyde . . . . . . . . . . . . . 270

369. Asarylaldehyd . . . . . . . . . . . . . . . . . . . . . 270

Ungesättigte Aldehyde. . . . . . . . . . . . . . . 273

370. o-Methoxyzimtaldehyd, o-Cumaraldehydmethyläther . . . . . . . . 273

c) Ketone . . . . . . . . . . . . . . . . . . . . . . . 275

1. Ketone der Benzolreihe, in denen Wasserstoff im Kern durch Hydroxyl bzw. Oxyalkyl substituiert ist . . . . 275

2. Ketone, welche Derivate von einwertigen Phenolen sind 275

371 u 372. o-Oxyacetophenon und ev. sein Alkyläther . . . . . . . . . 276

373. p-Oxyacetophenonderivat: Anisketon . . . . . . . . . . . . . 277

3. Keton der Benzolreihe, welches sich von einem zweiwertigen Phenol ableitet. . . . . . . . . . . . . . 279

374. Paeonol . . . . . . . . . . . . . . . . . . . . . . . . 279

d) Sauren der Benzolreihe, welche im Kern durch Hydroxyl bzw. Oxyalkyl usw. substituiert sind . . . . . . . . . 282

375. Salicylsäure . . . . . . . . . . . . . . . . . . . . . 282

376. Anissäure . . . . . . . . . . . . . . . . . . . . . . . 286

e) Laktone . . . . . . . . . . . . . . . . . . . . . . . 288

377. Dihydrocumarin, Melilotin . . . . . . . . . . . . . . . . 289

378. Cumarin . . . . . . . . . . . . . . . . . . . . . . . 291

p-Oxyzimtsäure $=$ p-Cumarsäure . . . . . . . . . . . . . . . . . . . . 297

379 u. 380 . Laktone von Dioxysäuren. Umbelliferon $=$ Oxycumarin . . . . . 297

Derivate von Trioxysäuren. Citrapten $=$ Limettin (Citronenölstearopten, Citronenölkampfer, Citropten) . . . . . . . . . . . . . . . 298

Bergapten . . . . . . . . . . . . . . . . . . . . . . . 301 
f) Ester von Säuren der Benzolreihe.

Ester von Säuren der Benzolreihe, die im Kern nicht durch Sauerstoff substituiert sind . . . . . . . . . . . . . . 303

Ester von gesättigten Săuren . . . . . . . . . . . 303

Ester der Benzoësäure. . . . . . . . . . . . . . . . . . . 303

Ester der Benzoësăure, deren alkohol. Bestandteil zur Methanreihe gehört . . . . . . . . . . . . . . . . . . . . 304

381. Benzoësăuremethylester . . . . . . . . . . . . . . . . . . 304

Ester der Benzoësäure mit ungesättigten aliphatischen Alkoholen

382. Geranylbenzoat

383. Linalylbenzoat

Peruviolbenzoat .

Ester der Benzoësäure mit Alkoholen der Benzolreihe . . . 309

384. Benzylbenzoat. . . . . . . . . . . . . . . . . . . . . . 309

385. Benzoësaurer Phenyläthylalkohol . . . . . . . . . . . . . . . 311

Ester der Phenylessigsäure . . . . . . . . . . . . . . . 311

386. Phenylessigsaurer Benzylester . . . . . . . . . . . . . . . . 313

387. Phenylessigsaurer Phenyläthylalkohl . . . . . . . . . . . . . 313

Ester von Säuren der Benzolreihe mit ungesattigter Seitenkette, die im Kern nicht durch Sauerstoff substituiert sind 313

Ester der Zimtsäure mit aliphatischen Alkoholen . . . . 314

388. Zimtsäuremethylester (Methylcinnamat). . . . . . . . . . . . . 314

389. Zimtsäureäthylester . . . . . . . . . . . . . . . . . . . . 316

390. Zimtsaures Peruviol (Zimtsăureperuvylester) . . . . . . . . . . . 317

Zimtsäureester, derer alkoholischer Bestandteil der Benzolreihe angehört. . . . . . . . . . . . . . . . . . . . 318

391. Zimtsäurebenzylester $=$ Cinnameïn . . . . . . . . . . . . . . 318

392. Zimtsäurephenylpropylester . . . . . . . . . . . . . . . . . 319

Zimtsaures Xanthoresinotannol . . . . . . . . . . . . . . . 320

Ester der Zimtsäure mit ungesattigten Alkohlen der Benzolreihe'. . . . . . . . . . . . . . . . . . 321

393. Styracin (Zimtsăurezimtester) . . . . . . . . . . . . . . . . 321

Ester von Säuren der Benzolreihe, welche im Kern durch Sauerstoff substituiert sind . . . . . . . . . . . . . . 323

Ester von gesättigten Säuren . . . . . . . . . . . . 323

Einwertig substituierte Benzoësäureabkömmlinge . . . . . . . . 323

394. Salicylsäuremethylester (Gaultheriaöl) . . . . . . . . . . . . 324

Ester der Salicylsäure mit Alkoholen der Benzolreihe . . . 335

395. Benzylsalicylat . . . . . . . . . . . . . . . . . . . . . 335

Ester von Dioxysäuren bzw. deren Derivaten . . . . . . . 335

396. Methylester der m-Methoxysalicylsäure . . . . . . . . . . . . 335

Ester der Veratrumsäure. . . . . . . . . . . . . . . . 336

397. Veratrumsäuremethylester . . . . . . . . . . . . . . . . . 336

398. Veratrumsäureäthylester . . . . . . . . . . . . . . . . . . 338

Ester von ungesättigten Säuren der Benzolreihe, die gleichzeitig im Kern durch Sauerstoff substituiert sind . . . 339

399. p-Oxymethylzimtsäureäthylester (p-Cumarsäuremethylester) . . . . . 339 
p-cumarsaures Xanthoresinotannol . . . . . . . . . . . . . . 340

Hydrocumarsaures Cumarin (melilotsaures Cumarin) . . . . . . . . 340

Ester unbekannter Konstitution. . . . . . . . . . . . . 341

Xanthorrhoeaharzöl (Xanthorrhoea hastilis) . . . . . . . . . . . 341

Tolubalsamöl (Toluifera Balsamum Mill.) . . . . . . . . . . . . . 341

g) S- und N-haltige Bestandteile ätherischeröle, soweit sie der

Benzolreihe angehören . . . . . . . . . . . . . . . . 341

$\mathrm{g}_{1}$ ) Nitrile der Benzolreihe (Ester der Blausäure) . . . . . . . 342

400. Phenylessigsäurenitril (Benzylcyanid) . . . . . . . . . . . . . 342

401. Nitril der Phenylpropionsäure. . . . . . . . . . . . . . . 344

402. Phenyloxyacetonitril (Mandelsäurenitril, Benzaldehydeyanhydrin) . . . $\mathbf{3 4 6}$

$\mathrm{g}_{2}$ ) Ester der Isothiocyansäure CS: NH; Senföle der Benzolreihe 347

403. Benzylsenföl . . . . . . . . . . . . . . . . . . . . . . 347

404. Phenyläthylsenföl . . . . . . . . . . . . . . . . . . . . . . . . 348

405. p-Oxybenzylsenföl . . . . . . . . . . . . . . . . . . . 350

$\mathrm{g}_{3}$ ) Basen der Benzolreihe . . . . . . . . . . . . . . . . 352

406. Anthranilsäuremethylester . . . . . . . . . . . . . . . . 353

407. N-Methylanthranilsäuremethylester . . . . . . . . . . . . $\quad$. 362

408. Damascenin . . . . . . . . . . . . . . . . . . . . . 364

\section{Heterocyklische Verbindungen.}

Heterocyklische Verbindungen mit einem Ringe . . . . . 368

Sauerstoff enthaltende Ringe . . . . . . . . . . . . . 368

409. Sylvan . . . . . . . . . . . . . . . . . . . . . . 368

410. Furfurol . . . . . . . . . . . . . . . . . . . . . . . 368

Furfuralkohol . . . . . . . . . . . . . . . . . . . . . . . . 372

411. Carlinaoxyd . . . . . . . . . . . . . . . . . . . . . 373

Stickstoffhaltige heterocyklische Verbindungen mit einem Ringe . . . . . . . . . . . . . . . . . . . 374

412. Pyrrol . . . . . . . . . . . . . . . . . . . . . . . . 374

413. N-alkyliertes Pyrrol . . . . . . . . . . . . . . . . . . . 375

Heterocyklische Verbindungen mit zwei Ringen. . . . . . 376

414. Indol . . . . . . . . . . . . . . . . . . . . . . . . . 377

415. Skatol . . . . . . . . . . . . . . . . . . . . . . . . . . 380

Anhang . . . . . . . . . . . . . . . . . . . . . . . . . . . . 381

1. N-haltige Bestandteile ätherischer Öle unbekannter Konstitution, die vielfach Basen sind . . . . . . . . . . . . . . . . . . . 381

2. Verbindungen unbekannter Zusammensetzung, die keinen Stickstoff enthalten

Übersicht über das gesamte Pflanzensystem unter besonderer Berücksichtigung der Pflanzen, die ätherische Öle liefern . . . . . 387

Autorenregister . . . . . . . . . . . . . . . . . . . . . . . 407

Sachregister . . . . . . . . . . . . . . . . . . . . . . . . . 443 